\title{
Experimental study of current sheet evolution in magnetic configurations with null-points and $\mathrm{X}$-lines
}

\author{
Anna G. Frank and Sergey Yu. Bogdanov \\ General Physics Institute of the Russian Academy of Sciences, Vavilov Str. 38, Moscow 117942, Russia
}

(Received June 20, 2000; Revised October 19, 2000; Accepted February 28, 2001)

\begin{abstract}
A review is presented on experimental research of current sheet formation in 3D magnetic configurations containing null-points and/or singular lines of the X-type. Formation of current sheets is revealed to occur in various 3D configurations, both with and without isolated magnetic null-points, specifically in configurations with X-lines. Local characteristics of 3D magnetic configuration define the parameters of the current sheet, which forms usually a twisted surface with an angular orientation determined by a local value of the transverse magnetic field gradient. A degree of plasma compression into the sheet decreases with a rise of longitudinal magnetic field component, displaying a transition to a behavior of uncompressible plasma. It is established that current sheet formation and magnetic reconnection processes can take place within a limited range of initial conditions, while a gradient of transverse magnetic field is the most important parameter.
\end{abstract}

\section{Introduction}

Magnetic reconnection in high-conductivity magnetized plasma pertains to the most important fundamental problems of modern plasma physics. At the same time reconnection is a basis for various phenomena of the flare-type, like solar and stellar flares, substormes in magnetospheres of the Earth and planets, sawtooth instabilities in tokamaks, rapid changes of the magnetic field structure in reversed field pinches, Zand $\theta$-pinches, etc. (Priest and Forbes, 2000). Magnetic reconnection phenomena occur at some discrete regions that separate magnetic fields of different, or opposite directions, and where electric currents of high density are concentrated. Localization of this region is generally determined by a position of a singular magnetic field line, since an electric field can exist along such lines in the plasma coordinate system (Syrovatskii, 1981). The best known examples of singular lines are the null-lines of 2D magnetic fields, and closed field lines in toroidal magnetic confinement systems.

Plasma dynamics in 2D magnetic fields with null-lines has been actively investigated for several decades. Experimentally it has been established that a planar current sheet (CS), which accumulates an excess magnetic energy, can be actually formed in a vicinity of the null-line (Frank, 1976; Stenzel and Gekelman, 1981). The magnetic field increased in $\cong 10$ times near the sheet surface, the electric current density $\cong 10 \mathrm{kA} / \mathrm{cm}^{2}$ peaked at the CS middle plane, the CS thickness $\cong 0.8 \mathrm{~cm}$ was about 10 times smaller than its width (Kirii et al., 1977). Plasma was rapidly compressed into the planar sheet: the electron density $N_{e}^{s h}=(1 \div 2) \cdot 10^{16} \mathrm{~cm}^{-3}$ exceeded both the initial and surrounding plasma densities in 10-15 times (Dreiden et al., 1977). It was revealed that

Copy right (C) The Society of Geomagnetism and Earth, Planetary and Space Sciences (SGEPSS); The Seismological Society of Japan; The Volcanological Society of Japan; The Geodetic Society of Japan; The Japanese Society for Planetary Sciences.
CS was rather stable, specifically the tearing-mode instability had no pronounced effect on the CS dynamics (Frank, 1989). Comparing with solar flares, one should treat the metastable CS as a pre-flare situation, while the flare itself should be associated with a fast destruction of CS (Syrovatskii, 1981). Principal features of flare phenomena: rapid change in the magnetic field topology, intensive plasma heating, generation of plasma flows, bursts of accelerated electrons, have been observed in the course of disruption of the laboratory produced CS (Frank, 1989). It was shown that interruption of the metastable stage and transition to the CS disruption were triggered by magnetic island formation inside CS followed by super-fast plasma heating, while the effect of plasma turbulence was of a secondary importance (Kirii et al., 1992; Frank et al., 1996b). Experimental results lead us to the conclusion that CS formation, which can be followed by its explosive disruption, represents the necessary condition for realization flare-type events, including gradual accumulation of magnetic energy, its fast release, plasma heating, mass ejection, and acceleration of charged particles.

Considerable recent attention has been focussed on 3D magnetic configurations, which are more general and much more typical for both astrophysical objects and laboratory plasma confinement devices. Theoretically CS formation has been predominantly associated with the presence of isolated magnetic null-points (Rosenau, 1979; Bulanov and Ol'shanetskii, 1984; Lau and Finn, 1990; Green, 1993), though it is well known that magnetic reconnection phenomena occur also in magnetic configurations containing no null-points, e.g. at tokamak plasmas (Kadomtsev, 1975). Experimental studies on magnetic reconnection in 3D configurations have been initiated in 1990s, and at present two experimental approaches are adopted. The first one is based on colliding of two initially produced magnetized plasmas with different orientations of magnetic fields; magnetic re- 
connection takes place at a region where two plasmas encounter (Yamada et al., 1990; Ono et al., 1993). The second approach is based on electric current excitation in the plasma placed in various 3D magnetic configurations, and reconnection phenomena occur if there are conditions for formation a distinguished region, like CS, where plasma electric current is concentrated (Frank et al., 1995).

In this paper a review is presented on experimental research of possibilities and conditions for CS formation in different 3D magnetic configurations containing some topological singularities: null-points and/or singular lines of the X-type.

\section{Experimental Device and Diagnostics}

The experimental device CS-3D (Three-Dimensional Current Sheet), which is employed for such investigations, operates in the following manner. An initial 3D magnetic configuration is formed at first, then plasma is produced in this configuration, and thereafter perturbations are excited giving rise to plasma flows and electric currents, which can result in CS appearance.

A basic principle for the formation of initial 3D magnetic configuration is a combination of two magnetic fields with different symmetry properties (Bulanov and Frank, 1992). One is 2D magnetic field of translational symmetry with two separatrix planes (SP): $(x=0),(y=0)$, and a null-line at the $z$-axis:

$$
\boldsymbol{B}_{q}=\left\{B_{x} ; B_{y} ; B_{z}\right\}=\{h \cdot x ;-h \cdot y ; 0\},
$$

here $h$ is the magnetic field gradient in the $(x, y)$ plane, $h \cong$ const. The second field is an axial-symmetric magnetic field with the symmetry-axis along the null-line. Near any point $(0,0, Z)$ at the $z$-axis this field can be presented in a form:

$$
\begin{aligned}
\boldsymbol{B}_{a s} & =\left\{B_{x} ; B_{y} ; B_{z}\right\} \\
& =B_{z}(Z)+h_{r}(Z) \cdot\{x ; y ;-2(z-Z)\},
\end{aligned}
$$

here $h_{r}(Z)$ is the radial field gradient. We make use of several types of axial-symmetric magnetic fields: the uniform $B_{z}$ field with $h_{r}(Z)=0$, the cusp-field with a null-point, where $B_{z}(Z)=0$, the non-uniform $B_{z}$-field without null-points.

A combination of two fields, (1) and (2), produces a novel 3D magnetic configuration:

$$
\begin{aligned}
\boldsymbol{B}_{\Sigma}=\boldsymbol{B}_{q}+ & \boldsymbol{B}_{a s}=B_{z}(Z)+h \cdot\{[1+\gamma(Z)] \cdot x ; \\
& -[1-\gamma(Z)] \cdot y ;-2 \gamma(Z) \cdot(z-Z)\},
\end{aligned}
$$

where a local value:

$$
\gamma(Z)=h_{r}(Z) / h
$$

is the ratio of two gradients, and it is an important parameter of the configuration, see below. Each field, (1) and (2), can be varied independently, providing formation of diversified 3D configurations with gradual transition from one configuration to another.

A basic diagram of the CS-3D device is shown in the Fig. 1. 2D magnetic field (1) with a null-line at the vacuum chamber axis is formed by a system of straight external conductors. Four coils (A, B, C, D) with various directions and magnitudes of electric currents produce axial-symmetrical magnetic field. Both magnetic fields are quasi-steady with respect

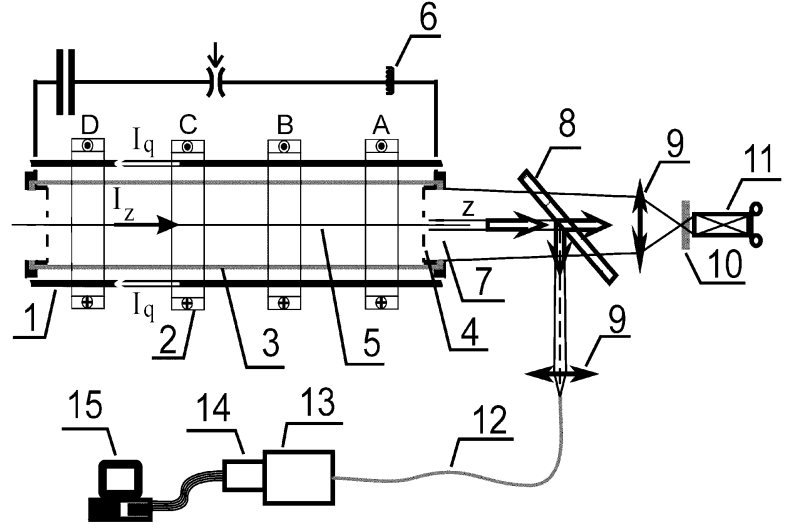

Fig. 1. Experimental device CS-3D: 1) straight conductors to produce 2D field (1); 2) four coils (A, B, C, D) to produce axial-symmetrical magnetic fields; 3) vacuum chamber $\emptyset=18 \mathrm{~cm}, l=100 \mathrm{~cm}$. 4) grid electrodes; 5) CS; 6) Rogowskii coil. 7) quartz windows; 8) 50\% mirror; 9) lenses; 10) interference filter; 11) frame-camera; 12) quartz optical fiber; 13) monochromator; 14) multi-channel optical registration system MORS-3; 15) PC.

to plasma processes. The vacuum chamber is filled with He gas, and the initial plasma is produced by $\theta$-discharge. Plasma electric current $\boldsymbol{I}_{z}$ is excited by applying a pulsed voltage between two electrodes moved apart at $60 \mathrm{~cm}$; maximum $\boldsymbol{I}_{z}$ value is varied within the range $\boldsymbol{I}_{z} \cong 40 \div 100 \mathrm{kA}$, its half-period is $T / 2=5 \mu \mathrm{s}$.

Some idea of plasma structures, which are formed in various 3D magnetic fields, can be gained from 2D distributions of plasma emission in the $(x, y)$ plane for different spectral lines. Plasma images (integral in $z$-direction) are recorded by an image intensifier combined with narrow-band interference filters (Bogdanov et al., 1994a). An analysis of the He II and He I spectral line profiles allows to deduce the electron density in CS (Buescher et al., 1999). Distributions of plasma electric current at different cross-sections can be obtained from magnetic measurements with a set of external magnetic probes. Subsequent data fitting by theoretical curves makes it possible to extract both CS width and angular orientation at each cross-section (Burilina et al., 1995).

\section{Current Sheet Formation in 3D Magnetic Con- figurations with Null-Points}

Experimental studies described below are concentrated on possibilities of CS formation in magnetic configurations with topological singularities, and configurations with null-points have been studied first (Bogdanov et al., 1994b). An initial configuration is produced by a combination of $2 \mathrm{D}$ field $\boldsymbol{B}_{q}$ (1) and a cusp field $\boldsymbol{B}_{c}$ with the SP $(z=0)$ and the null-point at $(0,0,0)$. The cusp field is generated using two coils, A and B, with electric currents of equal values and opposite directions; characteristics of this field are shown in the Fig. 2(a). In a vicinity of the null-point the magnetic field can be described as follows:

$$
\boldsymbol{B}_{c}=\left\{h_{r} \cdot x ; h_{r} \cdot y ;-2 h_{r} \cdot z\right\} .
$$

The superposition of (1) and ( $\left.2^{\prime}\right)$ builds up a new 3D configuration with a null point:

$$
\boldsymbol{B}_{\Sigma}=\boldsymbol{B}_{q}+\boldsymbol{B}_{c}=h\{(1+\gamma) \cdot x ;-(1-\gamma) \cdot y ;-2 \gamma \cdot z\}
$$



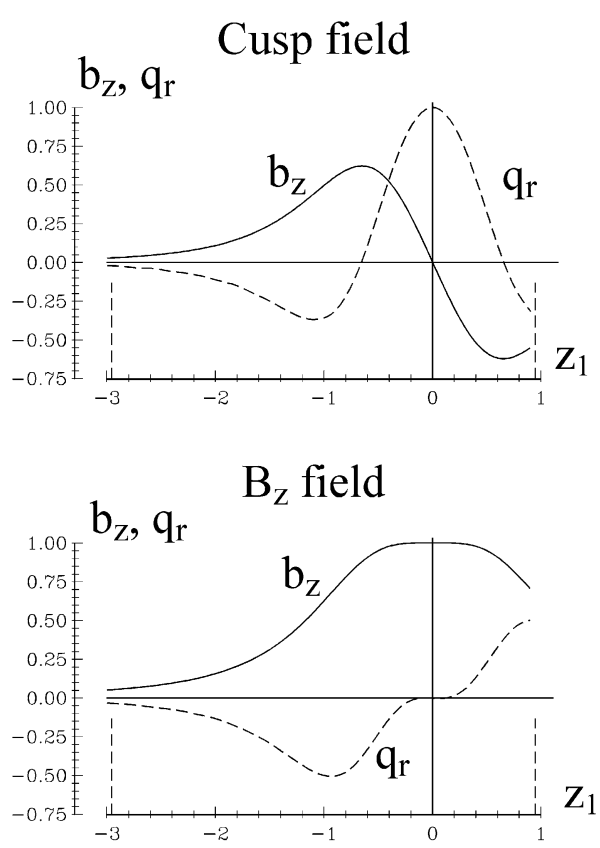

Fig. 2. $B_{z}$-component and radial field gradient hr of two axial-symmetrical magnetic fields: (a) the cusp-field with a null-point produced by two coils (A and B); (b) the field without null-point produced by Helmholtz-coils (A and B). $b_{z}=B_{z}(0,0, z) / B_{z}^{\max } ; q_{r}=h_{r}(0,0, z) / h_{r}^{\max } ; z_{1}=z / R_{c}$; $R_{c}=15.6 \mathrm{~cm}$.

here $\gamma=h_{r} / h$. By varying the $\gamma$-parameter it is possible to change significantly the structure of the magnetic field $\left(3^{\prime}\right)$, including the change in the field derivatives in different directions, and SP orientation (Frank, 1999).

It is well known that excitation of electric current along the null-line of 2D magnetic field (1) results in formation of a planar CS and efficient plasma compression into this sheet. 2D image of such plasma makes up a thin horizontal stripe extended along $x_{0}$-axis at the $(x, y)$ plane (Fig. 3(a), Bogdanov et al., 1994a). At 3D field containing a null-point the image becomes more complicated: the horizontal stripe is accompanied by an additional thin stripe, which is tilted at an angle $\alpha$ with respect to the axis $x_{0}$ (Fig. 3(b), Bogdanov et al., 1994b). The image indicates that a sheet arises also at $3 \mathrm{D}$ configuration, yet at some region the sheet orientation deflects significantly from its orientation in 2D field. It would appear reasonable to have a maximal sheet deflection near the null-point, at the plane $(z=0)$. Measurements by a set of magnetic probes make it evident that electric current distribution assumes really a sheet-shape at this plane; moreover, the CS orientation is found to be very close to the angle $\alpha$. Thus the conclusion, which we derive on the base of plasma images, is actually confirmed, and the results of both diagnostics coincide perfectly (Fig. 4(a)).

If the radial gradient $h_{r}$ of the cusp field $\left(2^{\prime}\right)$ was negative, then we obtain the reverse direction of sheet tilting at the summary configuration ( $\left.3^{\prime}\right)$, i.e. the angle $\alpha$ becomes negative (Fig. 3(c)). It should be stressed that the CS plane near the null-point assumes always an intermediate angular position between 2D case $(\alpha=0)$ and the SP orientation at the initial 3D configuration: $\alpha^{\mathrm{SP}}=45^{\circ}$ (Figs. 3(b), (c)).

Being rather flexible, the device CS-3D allows employ a

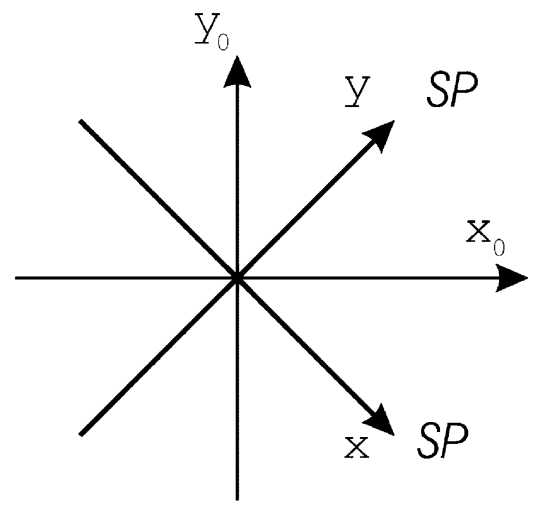

b

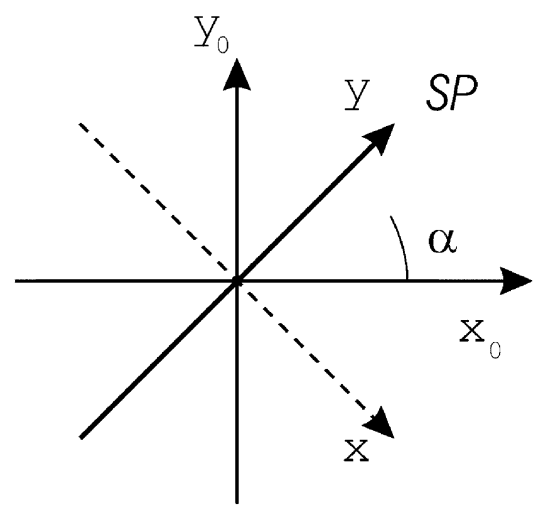

C

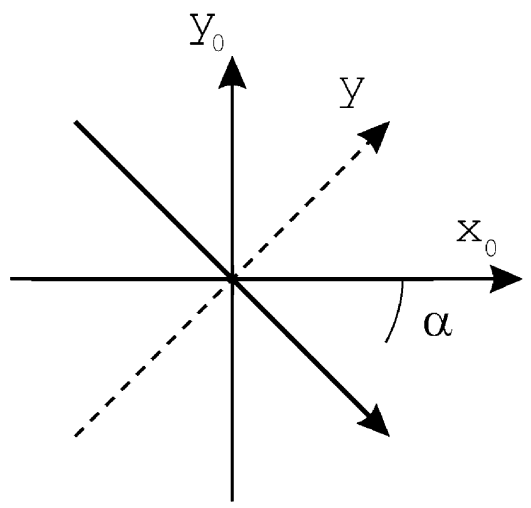

Fig. 3. Schematic plasma images in $(x, y)$ plane, $z$-integrated. (a) 2D field (1), $h=0.2 \mathrm{kG} / \mathrm{cm}$. (b) and (c) 3D magnetic fields with null-points, $h=0.2 \mathrm{kG} / \mathrm{cm}$; (b) $h_{r}=0.13 \mathrm{kG} / \mathrm{cm}, \gamma=h_{r} / h>0, \alpha=30^{\circ}$. (c) $h_{r}=-0.13 \mathrm{kG} / \mathrm{cm}, \gamma<0 ; \alpha=-30^{\circ}$.

various combinations of gradients, $h$ and $h_{r}$, which characterize magnetic fields $(1)$ and $\left(2^{\prime}\right)$. In this way a wide range of $3 \mathrm{D}$ magnetic configurations with null-points $\left(3^{\prime}\right)$ can be produced. It is impressive that CS formation takes place throughout all these configurations, as follows from both plasma images and magnetic measurements. At the same time, a gradual change occurs in the CS angular orientation at progressive deformation of a configuration. Thus, an increase in $h_{r}$ at fixed $h$ causes an increase in the angle $\alpha$ (Fig. 4(a)), contrary to $\alpha$ decrease caused by an increase in $h$ at fixed $h_{r}$ (Fig. 4(b)), curve I (Frank et al., 1995). A 

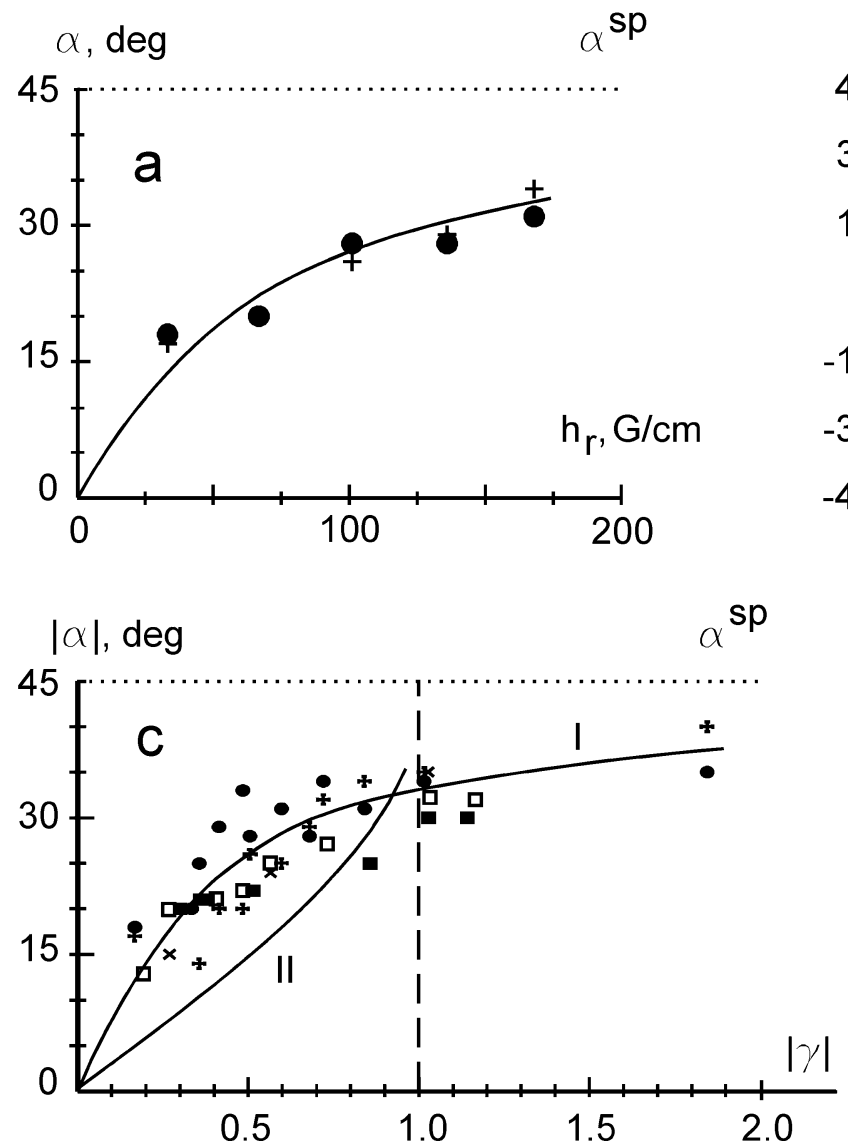

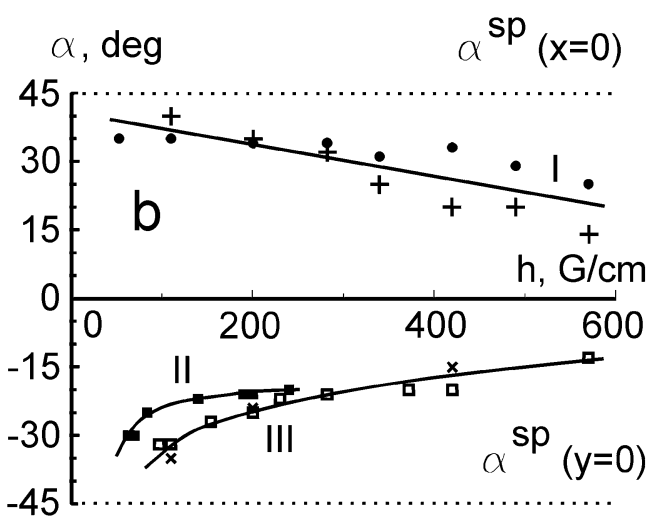

Fig. 4. CS angular orientation $\alpha$ in 3D magnetic configurations as a function of: (a) $h_{r}$, (b) $h$, and (c) local $|\gamma|$-value, curve I. Points A, B, C correspond to configurations with null-points; points D, E-to configurations without null-points. Data obtained from plasma images (A, E) and from magnetic measurements (B, C, D): (B) section $z_{1}=0$, as shown in the Fig. 2; (C, D) section $\left(z_{1}=-1\right)$. Angular orientation of the perpendicular dropped from the null-point to the vacuum magnetic field lines at the section $z_{1}=0$, (c) curve II.

large body of similar data makes us conclude that CS angular orientation near a magnetic null-point is dictated by the parameter $\gamma$ (4) (Fig. 4(c), points A, B), while $\gamma$ may be changed by varying either $h$ or $h_{r}$.

We observe that CS angular orientation follows both the value and the sign of $\gamma$, and CS is universally tilted towards SP as $\gamma$ increases, but do not reach SP, so that $\alpha<\alpha^{\mathrm{SP}}$ (Fig. 4(c), curve I). It turns out that CS angular position in a vicinity of the null-point is approximately normal to the magnetic field lines of the initial 3D configuration at the plane $(z=0)$ (Fig. 4(c), curve II), (Bogdanov et al., 1994b). Notice that CS formation is also observed experimentally under conditions $1 \leq \gamma \leq 2$ (Fig. 4(c)), i.e. when plasma electric current $I_{z}$ is perpendicular to the SP $(z=0)$. Thus it has been established experimentally for the first time that CS formation really takes place at $3 \mathrm{D}$ magnetic configurations near isolated null-points.

\section{Current Sheet Formation in 3D Non-Uniform Magnetic Fields without Null-Points}

A vicinity of a null-point forms only a small part of 3D magnetic configuration (Fig. 2(a)), so a question appears how far from a null-point the formation of CS can occur? On the base of magnetic measurements we can conclude that electric current acquires a form of a sheet in every cross section of the CS-3D device. At the same time the CS angular orientation differs both in direction and in magnitude from one section to another (Fig. 4(b) (curves I, II), Frank et al., 1995). Taking into account the $z$-dependence of $h_{r}$ (Fig. 2(a)), we obtain that CS local angular position follows the local value of the parameter $\gamma(Z)$ (Fig. 4(c), points C) by analogy with a region near the null-point. Note that far away from the null-point $C S$ is formed in a presence of relatively strong $B_{z}$-field, e.g. at the $z_{1} \cong-1$ cross-section (Fig. 2(a)). Thus, the distribution of the electric current assumes the sheet shape everywhere at $3 \mathrm{D}$ configuration with a null-point, and CS constitutes a twisted surface (Fig. 5).

To exclude a possible influence of null-points on CS formation we produce $3 \mathrm{D}$ configurations by superposition the 2D field (1) and the axial-symmetrical non-uniform magnetic field without null-point. The field is generated using the same two coils, A and B at the Fig. 1, with electric currents of equal values and directions, i.e. by a system of Helmholtz coils. Characteristics of this field are shown in the Fig. 2(b). Analysis of plasma images as well as magnetic measurements in several cross-sections allows to state that CS formation occurs also in magnetic fields without null-points. Angular sheet orientation in every cross-section follows again the local value of the parameter $\gamma(Z)$ (Fig. 4(b), curve III; Fig. 4(c), points $\mathrm{D}, \mathrm{E})$. At the cross-section $z_{1} \cong 0$ the sheet is observed to be similar to the sheet produced in $2 \mathrm{D}$ field (1) 


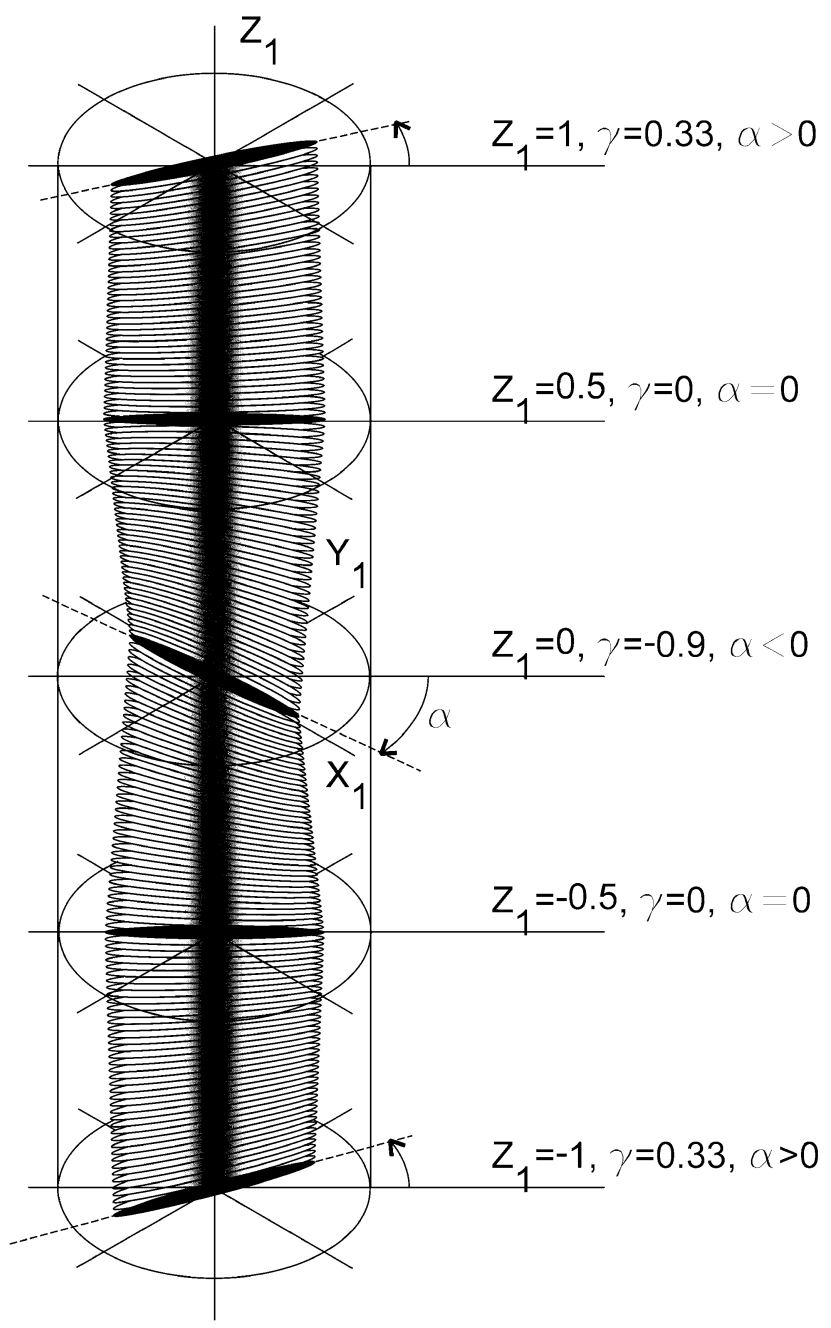

Fig. 5. Twisted current sheet formed in 3D magnetic field with a null-point. The $z_{1}$-scale corresponds to the Fig. 2(a), the null-point is at the plane $\left(z_{1}=0\right)$.

(Fig. 3(a)). Hence it follows that rather strong $B_{z}$-field does not prevent the CS formation.

Thus it is revealed that CS formation is a universal phenomenon for plasma dynamics in 3D non-uniform magnetic fields, both containing isolated null-points and without them. As a rule, 3D CS is a twisted surface, while its angular orientation at any cross-section is determined by local value of the transverse gradient of the initial 3D magnetic field (Frank et al., 1996a; Frank, 1999).

\section{Current Sheets in Magnetic Configurations with Singular X-Lines}

The effect of $B_{z}$-field on possibilities of CS formation and its parameters makes itself evident in 3D configurations containing singular lines of the X-type with uniform longitudinal $B_{z}$-component:

$$
\boldsymbol{B}_{\Sigma}=\left\{h x ;-h y ; B_{z}\right\}
$$

here $\partial B_{z} / \partial z=0$, and the magnetic field components are $z$ independent. It should be noted that X-type singular line can be classified as a more general structural element of magnetic field than null-point or null-line. The null-line is the X- line without $B_{z}$-component, and in configurations with nullpoints, the singular X-line is present both in the region of zero magnetic field and far away from this region.

The main attention is focused on quantitative characteristics of magnetic configurations, in which CS can be formed, while magnetic field (5) is produced under different combinations of $h$ and $B_{z}$. 2D images reveal some peculiarities of plasma structures arising after electric current $I_{z}$ is generated along the X-line (Fig. 6, Bogdanov et al., 2000). The images can be divided in two groups: frames $1,2,5,6$ correspond to formation of planar CS, while frames 3, 4, 7, 8 demonstrate formation of sheet-like double-piece structures separated by a slopping split.

Though $B_{z}$ exceeds the transverse field $\left|\boldsymbol{B}_{\perp}\right|=h \times|r|$ in the most part of plasma volume, the images from the first group are similar to those observed when CS is formed in 2D field (1) with $B_{z}=0$. The images have almost the same shape in the HeII and HeI spectral lines, except burning out of $\mathrm{HeI}$ in the sheet middle plane. Magnetic measurements confirm the formation of planar CS in each cross-section ( $z=$ const $)$. At the same time plasma density in CS turns out to be 2-3 times lower as compared with 2D CS, indicating that $B_{z}$-component cause a decrease in plasma compression (Frank et al., 1998). The effect relates apparently to $B_{z}$ enhancement inside CS due to magnetic field transport by plasma flows, while an influence of $B_{z}$-field can be analogous to an additional gas-kinetic pressure (Syrovatskii,

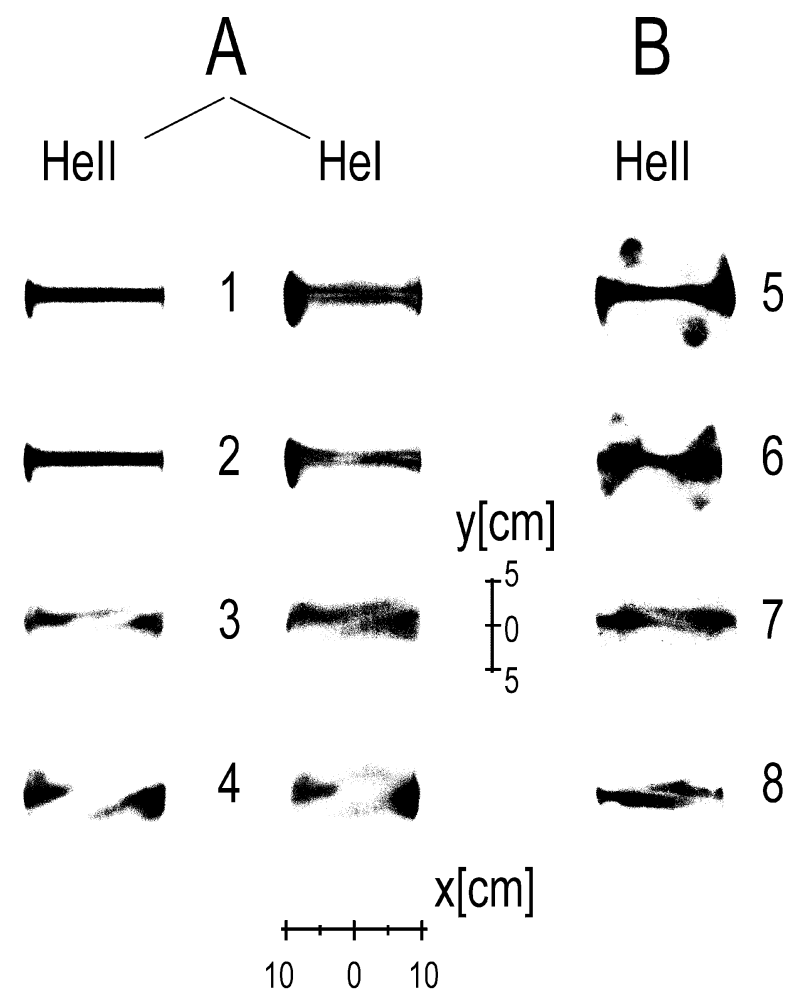

Fig. 6. Plasma images in the HeII 468.6- and HeI 587.6-nm spectral lines registered in magnetic configurations with X-line under different conditions. (A) $B_{z}=4.3 \mathrm{kG}, I_{z}^{\max } \cong 100 \mathrm{kA}, t \cong 2.5 \mu \mathrm{s}$, and different transverse gradients $h$ : (1) $h=0.57$, (2) 0.42 , (3) 0.28 , and (4) 0.2 $\mathrm{kG} / \mathrm{cm}$. (B) $h=0.2 \mathrm{kG} / \mathrm{cm}, I_{z}^{\max } \cong 40 \mathrm{kA}, t \cong 2 \mu \mathrm{s}$, and different magnitudes of longitudinal magnetic field component $B_{z}$ : (5) $B_{z}=0$, (6) 1.4, (7) 2.9 , and (8) $5.7 \mathrm{kG}$. 


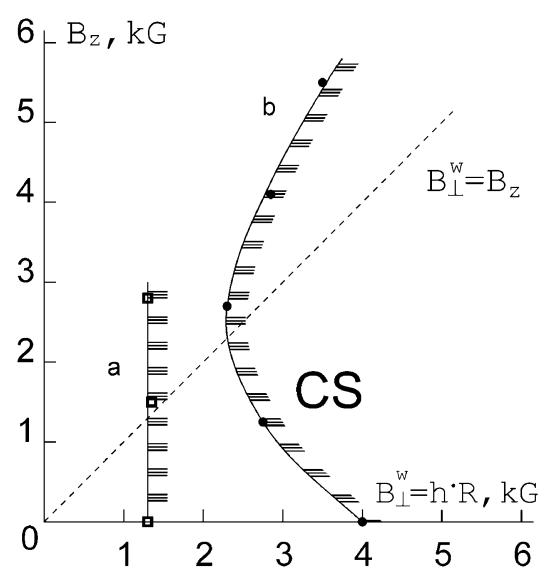

Fig. 7. The ranges of parameters of the magnetic configurations with X-line: $\boldsymbol{B}_{\Sigma}=\left\{h x ;-h y ; B_{z}\right\}$, where current sheet formation occurs. $B_{\perp}^{W}=h \times R_{k}$-transverse magnetic field at the plasma boundary. $B_{z}$-longitudinal magnetic field component along the X-line. $I_{z}=50$ $\mathrm{kA}$; He-pressure: $p_{0}=60 \mathrm{mTorr}(\mathrm{a}) ; p_{0}=300 \mathrm{mTorr}(\mathrm{b})$.

1981). Extra $B_{z}$-field inside $C S$ is estimated as $\approx 90 \%$ of the initial $B_{z}$-value, and the total $B_{z}$-field peaks at $\approx 8 \mathrm{kG}$ (Bogdanov et al., 2000). Thus we observe a gradual transition to the dynamics of uncompressible plasma.

As follows from the Fig. 6, formation of plasma structures, which differ from planar sheet, occurs as the longitudinal magnetic field component $B_{z}$ increases and the transverse gradient $h$ decreases. When $B_{z}=4.3 \mathrm{kG}$ is kept constant, Fig. 6 (column A), the sheet formation becomes impossible under $h<0.28 \mathrm{kG} / \mathrm{cm}$, which corresponds to $B_{z} / h>15.3$ $\mathrm{cm}$. By contrast, when the gradient is kept constant, $h=0.2$ $\mathrm{kG} / \mathrm{cm}$ (column B), the sheet is not observed under $B_{z}$. > $2.9 \mathrm{kG}\left(B_{z} / h>14.5 \mathrm{~cm}\right)$. These results indicate that CS formation takes place only under conditions when $B_{z} / h$ do not exceed a certain critical value: $B_{z} / h \approx 15 \mathrm{~cm}$ for the present experiment, being presumably related to geometrical factors. Plasma structures for $B_{z} / h>15 \mathrm{~cm}$ develop in the sheet-like form but with a slopping split that divides the structure in two parts (Fig. 6 (frames 3, 4, 7, 8)). Plasma emission in the both HeII and HeI spectral lines is absent inside the split, pointing up to a local minimum of plasma density in this region.

Generally speaking we can draw a demarcation line in the plane $\left(B_{z}, B_{\perp}^{W}=h \times R_{k}\right)$, which separates two regions for each set of initial conditions (Fig. 7). CS formation takes place to the right of both lines $(a, b)$, and there arise another plasma structures to the left of the lines. The line position, in turn, depends on the initial plasma density and electric current. Experimental data make us conclude that the most important parameter, which defines a possibility of CS formation, is the transverse magnetic field gradient $h$. Thus it is shown for the first time that CS formation and, consequently, magnetic reconnection phenomena can take place within a limited range of initial conditions.

\section{Summary}

The review of experimental research is presented on possibilities of CS formation in different 3D magnetic configurations with null-points and/or singular lines of the X-type.
It is revealed that CS can appear in various 3D configurations, both with and without isolated magnetic null-points, specifically in configurations with X-lines. Local characteristics of 3D magnetic configuration define the parameters of CS, which forms usually a twisted surface with an angular orientation determined by local value of the transverse magnetic field gradient $h$.

Formation of planar CS takes place in 3D magnetic fields with X-lines, i.e. in a presence of fairly strong longitudinal magnetic field component directed along the X-line, including the condition $B_{z}>B_{\perp}$ everywhere at the plasma volume. A degree of plasma compression into the sheet decreases with a rise of the longitudinal component, displaying a transition to a behavior of uncompressible plasma. However if $B_{z} / h$ exceeds a critical value, an appearance of planar CS becomes impossible; instead there is formed a sheet-like double-piece plasma with a cavity in the central region.

It is established for the first time that CS formation and magnetic reconnection processes can take place within a limited range of initial conditions, while the transverse magnetic field gradient $h$ is the most important parameter.

Acknowledgments. The authors thank V. B. Burilina, N. P. Kyrie, and V. S. Markov for assistance in experiment and discussions. This work is supported by the Russian Foundation for Basic Research, Project N 99-02-18351, and partly (for A. F.) by the Ministry of Education of Japan.

\section{References}

Bogdanov, S. Yu., Yu. F. Bondar, V. B. Burilina et al., Spatiotemporal evolution of emission in different spectral lines from a current-sheet plasma, Tech. Phys., 39, 877-881, 1994a.

Bogdanov, S. Yu., V. B. Burilina, V. S. Markov, and A. G. Frank, Formation of current sheets in 3D magnetic fields with a null point, JETP Lett., 59 , 537-541, 1994b.

Bogdanov, S. Yu., N. P. Kyrie, V. S. Markov, and A. G. Frank, Current sheets in magnetic configurations with singular X-lines, JETP Lett., 71, 53-58, 2000

Buecsher, St., N. P. Kyrie, H.-J. Kunze, and A. G. Frank, Spectroscopic measurements of the electron density in a current sheet produced under high-pressure helium filling, Plasma Phys. Reports, 25, 164-169, 1999.

Bulanov, S. V. and M. A. Ol'shanetskii, On the magnetic collapse near the zero points of the magnetic field, Phys. Lett., 100A, 35-38, 1984.

Bulanov, S. V. and A. G. Frank, About one approach to the experimental study of magnetic reconnection in three-dimensional magnetic configurations, Sov. J. Plasma Phys., 18, 795-799, 1992.

Burilina, V. B., V. S. Markov, and A. G. Frank, Determination of currentlayer parameters in three-dimensional magnetic-field configurations based on magnetic measurements, Plasma Phys. Reports, 21, 33-37, 1995.

Dreiden, G. V., N. P. Kyrie, V. S. Markov et al., Holographic-interference study of the plasma density distribution in a current sheet, Sov. J. Plasma Phys., 3, 26-31, 1977.

Frank, A. G., Experimental study of the conditions for the appearance of a neutral current sheet in a plasma. Some characteristics of the sheet, in Neutral Current Sheets in Plasmas, Proc. P.N. Lebedev Phys. Inst. Acad. Sci. USSR, edited by N. G. Basov, Consultant Bureau, N.Y., 74, 107-160, 1976.

Frank, A. G., Formation, evolution, and explosive disruption of current sheets in plasma, in Plasma Physics and Plasma Electronics, edited by L. M. Kovrizhnykh, pp. 131-169, Nova Science Publishers, Commack, N.Y., 1989.

Frank, A. G., Magnetic reconnection and current sheet formation in 3D magnetic configurations, Plasma Physics and Control. Fusion, 41 Suppl. 3A, A687-A697, 1999.

Frank, A. G., S. Yu. Bogdanov, and V. B. Burilina, Quasi-one-dimensional current sheets in three-dimensional magnetic configurations, Bull. Russ. Acad. of Sciences (Physics), 59, 1331-1341, 1995.

Frank, A. G., S. Yu. Bogdanov, V. B. Burilina, and N. P. Kyrie, Magnetic 
Reconnection through the Current Sheet as the Universal Process for Plasma Dynamics in Nonuniform Magnetic Fields, Proc. Intern. Conf. on Plasma Phys., Nagoya, Japan, 1, 506-509, 1996a.

Frank, A. G., V. P. Gavrilenko, Ya. O. Ispolatov et al., Anomalous electric fields inside a dense plasma of a current sheet, Contrib. Plasma Phys., 36, 667-678, 1996b.

Frank, A. G., S. Yu. Bogdanov, N. P. Kyrie, and V. S. Markov, Experiments on Magnetic Reconnection in 3D Configurations with X-Line, Proc. 1998 ICPP \& 25th EPS Conf. on Contr. Fusion and Plasma Physics, Praha, Czech Republic, ECA, 22C, 2196-2199, 1998.

Green, J. M., Reconnection of vorticity lines and magnetic lines, Phys. Fluids B, 5, 2355-2362, 1993.

Kadomtsev, B. B., Disruptive instability in tokamaks, Sov. J. Plasma Phys., 1, 389-391, 1975

Kirii, N. P., V. S. Markov, A. G. Frank, and A. Z. Khodzhaev, Rapid change in the structure of the magnetic field of a current sheet, Sov. J. Plasma Phys., 3, 303-306, 1977.

Kirii, N. P., V. S. Markov, and A. G. Frank, Local pulsed plasma heating and destruction of a current sheet, JETP Letts., 56, 82-87, 1992.

Lau, Y.-T. and J. M. Finn, Three-dimensional kinematic reconnection in the presence of field nulls and closed field lines, Astrophys. J., 350, 672-691, 1990

Ono, Y., A. Morita, M. Katsurai, and M. Yamada, Experimental investigation of three-dimensional magnetic reconnection by use of two colliding spheromaks, Phys. Fluids B, 5, 3691-3701, 1993.

Priest, E. R. and T. Forbes, Magnetic Reconnection. MHD Theory and Applications, 600 pp., Cambridge University Press, 2000

Rosenau, P., Three-dimensional flow with neutral-points, Phys. Fluids, 22, 849-858, 1979.

Stenzel, R. L. and W. Gekelman, Magnetic field line reconnection experiments. 1. Field topologies, J. Geophys. Res., 86, 649-658, 1981.

Syrovatskii, S. I., Pinch sheets and reconnection in astrophysics, Annu. Rev. Astron. Astrophys., 19, 163-229, 1981.

Yamada, M., Y. Ono, A. Hayakawa et al., magnetic reconnection of plasma toroids with cohelicity and counterhelicity, Phys. Rev. Lett., 65, 721-724, 1990.

A. G. Frank (e-mail: annfrank@fpl.gpi.ru) and S. Yu. Bogdanov 